\title{
Comparative assessment of left atrial volume in healthy cats by two-dimensional and three-dimensional echocardiography
}

\author{
Janina Rauch $^{1 *} \mathbb{D}$, Michael Fehr ${ }^{1}$, Martin Beyerbach² and Stephan O. Hungerbuehler ${ }^{3}$
}

\begin{abstract}
Background: The left atrium $(L A)$ is an important prognostic parameter in cardiac pathologies of cats. Its size is currently measured in one-dimensional methods, while human medicine considers two- and three-dimensional echocardiography as standard. The objectives of this study were to compare monoplane, biplane, triplane and realtime three dimensional echocardiography for volumetric measurement of the left atrium in healthy cats and establish a reference interval for further studies on cats with heart disease. Additionally, the influence of age, sex and weight on left atrial volume (LAV) was tested.

Results: One dimensional monoplane Simpson method of discs (SMOD) in the right parasternal four chamber view (r4) and the left apical 2 chamber view (12) as well as biplane SMOD had no significant difference for left atrial maximum volume (LAMax). They can be used as equivalent in future studies and one common reference range was set up $(1.96 \pm 0.54 \mathrm{ml})$. Those three methods produced significantly higher volumes than triplane echocardiography (RTTPE) and real time three dimensional echocardiography (RT3DE) using TomTec ${ }^{\oplus}$ software. LA volumetry with RTTPE and RT3DE-TomTec ${ }^{\text {TM }}$ was more feasible than expected, but low RT3DE image quality was the main reason for excluding patients. Neither age nor weight had an influence on LA volume in healthy cats. Male $L A V$ results were only slightly, but in $2 \mathrm{D}$ and RTTPE significantly higher than those of female cats with a range of + $10.46 \%$ to $+19.58 \%$.
\end{abstract}

Conclusions: Monoplane, biplane, triplane and real-time three dimensional echocardiography were feasible for LA volumetry in healthy cats and showed acceptable intra- and interobserver variability. One common LAMax reference range for monoplane 14,12 and biplane SMOD was set up. Raw data can be used for LA volumes and does not need to be correlated with the cat's weight or age. Male cats have only slightly but significantly larger atria than females in 2D and RTTPE. Therefore, under reservation, also sex related limit values were defined.

Keywords: Cat, Left atrium, Volumetry, RT3DE, RTTPE, LA, 2D, 3D

\section{Background}

The left atrium (LA) is an important prognostic parameter in cardiac pathologies of cats [1-3]. LA size significantly increases with progressing severity of left sided cardiomyopathies [1, 3-7]. Furthermore, an expansion

\footnotetext{
* Correspondence: nina.rauch@hotmail.com

${ }^{1}$ Small Animal Clinic, University of Veterinary Medicine Hannover, Foundation, Bünteweg 9, D-30559 Hannover, Germany

Full list of author information is available at the end of the article
}

of LA leads to decelerated blood flow in the LA and in the left atrial appendage (LAA). This causes an increased risk for life threatening aortic thromboembolism in cats with dilatation of the LA $[8,9]$. Left sided congestive heart failure that is detected too late can also lead to secondary pulmonary hypertension in cats [10].

Radiography and ECG are specific, but insensitive methods for evaluation of LA enlargement [11]. CMRI is golden standard for the measurement of LA in human 
medicine because it captures the most realistic asymmetrical structure, but the requirement of general anesthesia and high costs make MRI an unsuitable standard examination method in cats [12]. No comparable studies have been performed. The best non-invasive way to determine LA size and function in awake cats is via echocardiography [13]. Currently, one-dimensional (1D) diameter measurements are most commonly used in cats because they are simple and fast to perform [13]. Furthermore, the ratio between the LA diameter and the diameter of the aortic valve (LA/AO) is used to detect atrial dilatation [1, 13-21]. Recently, some authors have reported that pulmonary vein to pulmonary artery ratio variables were better factors than LA/AO for identifying cats with congestive heart failure [22].

The common disadvantage of these methods is the evaluation of a three-dimensional (3D) structure based on a single diameter. Consequently, there is a risk that a dilatation, which can occur in all 3 dimensions, will not be detected.

The quantification of the LA area and ratios of LA area and circumference measurements with the aorta are not commonly used, because mono- and multiplane volume measurements have shown the potential to be more reliable for early diagnosis of LA enlargement in cats, dogs and humans $[5,9,14,23-26]$. The two most common techniques are area-length $(\mathrm{A} / \mathrm{L})$ and Simpson
Method of Discs (SMOD). Each method can be used to calculate monoplane volume or biplane volume.

Monoplane SMOD measurement in the $\mathrm{r} 4$ and 14 plane was used for evaluation of LA size in two studies before and was proven to be a promising echocardiographic method for LA volumetry $[5,26]$.

The American Society of Echocardiography and the European Association of Cardiovascular Imaging recommend biplane SMOD over A/L, because it is based on less geometric assumptions [27]. There are no studies comparing $\mathrm{A} / \mathrm{L}$ and SMOD or monoplane and biplane methods for LA volumetry in cats.

Triplane volumetric examination (RTTPE) is a threedimensional method using 3 planes in a $60^{\circ}$ angle at the same time. In left ventricle volumetry (LVV), RTTPE was proven to be more accurate and faster than SMOD when compared to gold standard cMRI in humans and dogs $[28,29]$. There are no studies for evaluation of LA using RTTPE in veterinary medicine.

Real time three dimensional echocardiography (RT3DE) is the most modern ultrasound technique and generates a dynamic three dimensional volume block of the heart based on endocardial border detection. In human medicine, RT3DE using the software TomTec ${ }^{\circ}$ provides results that underestimate cMRI calculations less than 2DE, as they capture asymmetrical LA expansion in contrast to $2 \mathrm{DE}[23,30,31]$. Until now, there is very little experience with three dimensional LA volumetry in

Table 1 Overview of left atrial volumes (LAV Excluding Appendage): left atrial volumes in healthy cats $(n=50)$ measured with different methods. Data is presented as mean \pm standard deviation in millilitre $(\mathrm{ml})$ and as $95 \%$ prediction interval in the brackets (ml)

\begin{tabular}{|c|c|c|c|c|}
\hline & LAMax & LAMin & EF & SV \\
\hline \multirow{2}{*}{ R4AL } & $2.08 \pm 0.61$ & $0.79 \pm 0.27$ & $61.75 \pm 7.84$ & $1.30 \pm 0.43$ \\
& $(1.91-2.25)$ & $(0.71-0.86)$ & $(59.52-63.98)$ & $(1.17-1.42)$ \\
\hline \multirow{2}{*}{ R4 } & $1.95 \pm 0.56$ & $0.74 \pm 0.25$ & $61.84 \pm 7.42$ & $1.21 \pm 0.39$ \\
& $(1.79-2.11)$ & $(0.67-0.81)$ & $(59.74-63.96)$ & $(1.10-1.32)$ \\
\hline \multirow{2}{*}{ L2 } & $1.98 \pm 0.63$ & $0.77 \pm 0.24$ & $60.93 \pm 8.26$ & $1.22 \pm 0.44$ \\
& $(1.82-2.15)$ & $(0.70-0.83)$ & $(58.58-63.28)$ & $(1.10-0.34)$ \\
\hline \multirow{2}{*}{ L4 } & $1.77 \pm 0.49$ & $0.54 \pm 0.20$ & $69.77 \pm 5.93$ & $1.23 \pm 0.34$ \\
& $(1.63-1.91)$ & $(0.48-0.60)$ & $(68.08-71.45)$ & $(1.14-1.33)$ \\
\hline \multirow{2}{*}{ Biplane } & $1.93 \pm 0.50$ & $0.65 \pm 0.21$ & $65.62 \pm 6.35$ & $1.27 \pm 0.36$ \\
& $(1.79-2.07)$ & $(0.60-0.72)$ & $(63.82-67.42)$ & $(1.46-1.67)$ \\
\hline \multirow{2}{*}{ RTTPE } & $1.74 \pm 0.52$ & $0.68 \pm 0.23$ & $60.17 \pm 8.96$ & $1.06 \pm 0.38$ \\
& $(1.59-1.89)$ & $(0.61-0.75)$ & $(57.62-62.71)$ & $(0.95-1.17)$ \\
\hline \multirow{2}{*}{ 4D-TomTec ${ }^{\text {TM }}$} & $1.61 \pm 0.52$ & $0.68 \pm 0.32$ & $57.41 \pm 13.60$ & $0.93 \pm 0.38$ \\
& $(1.47-1.76)$ & $(0.59-0.78)$ & $(53.55-61.28)$ & $(0.82-1.04)$ \\
\hline
\end{tabular}

Abbreviations: Max Left atrial maximum volume, Min Left atrial minimal volume, EF Ejection Fraction, SV Stroke Volume, R4AL right parasternal 4 chamber view measured with 2D Area/Length method, R4 2D monoplane Simpson method of discs, L2 left apical two chamber view, L4 left apical four chamber view, Biplane L2 and L4 combined to biplane volumetry, RTTPE Real Time Triplane Echocardiography, 4D-TomTec ${ }^{\text {TM }}$ analysing software 
Table 2 Overview of P-Values for all left atrial (LA) measurement methods

\begin{tabular}{|c|c|c|c|c|}
\hline Methods & LAMax & LAMin & $\mathrm{EF}$ & SV \\
\hline R4 vs R4AL & $<0.0001$ & $<0.0001$ & 0.5814 & $<0.0001$ \\
\hline R4 vs L2 & 0.6690 & 0.3364 & 0.5530 & 0.9399 \\
\hline R4 vs L4 & 0.0126 & $<0.0001$ & $<0.0001$ & 0.7380 \\
\hline R4 vs Biplane & 0.7009 & 0.0045 & 0.0026 & 0.3092 \\
\hline R4 vs RTTPE & 0.0042 & 0.0914 & 0.3076 & 0.0112 \\
\hline R4 vs 4DTomTec ${ }^{T M}$ & 0.0001 & 0.2473 & 0.0315 & $<0.0001$ \\
\hline L2 vs L4 & 0.0021 & $<0.0001$ & $<0.0001$ & 0.3003 \\
\hline L2 vs Biplane & 0.0883 & $<0.0001$ & $<0.0001$ & 0.0541 \\
\hline L2 vs RTTPE & $<0.0001$ & 0.0006 & 0.5378 & 0.0005 \\
\hline L2 vs 4DTomTec ${ }^{\mathrm{TM}}$ & $<0.0001$ & 0.0869 & 0.1030 & $<0.0001$ \\
\hline L4 vs Biplane & $<0.0001$ & $<0.0001$ & $<0.0001$ & 0.1972 \\
\hline L4 vs RTTPE & 0.5752 & $<0.0001$ & $<0.0001$ & 0.0004 \\
\hline L4 vs 4DTomTec ${ }^{\mathrm{TM}}$ & 0.0185 & 0.0007 & $<0.0001$ & $<0.0001$ \\
\hline Biplane vs RTTPE & $<0.0001$ & 0.3309 & $<0.0001$ & $<0.0001$ \\
\hline Biplane vs 4DTomTec ${ }^{\mathrm{TM}}$ & $<0.0001$ & 0.5469 & $<0.0001$ & $<0.0001$ \\
\hline RTTPE vs 4DTomTec ${ }^{\mathrm{TM}}$ & 0.0135 & 0.9260 & 0.1949 & 0.0044 \\
\hline
\end{tabular}

Comparison of left atrial maximum and minimum volumes (LAMax/LAMin), ejection fraction (EF) and stroke volume (SV) in 50 healthy cats measured with 7 echocardiographic methods: right parasternal 4 chamber view measured with 2D Area/Length method (R4AL) and 2D monoplane Simpson method of discs (R4), left apical two chamber view (L2) and four chamber view (L4) measured with 2D monoplane Simpson method of discs, L2 and L4 combined to biplane volumetry (Biplane), Real Time Triplane Echocardiography (RTTPE), 4D-TomTec ${ }^{T M}$ analysing software. Mean differences between the measurementtechniques were evaluated with a t-Test. $P>0.05$ was evaluated as significant similarity and marked as bold letters

veterinary medicine. All available studies have been performed in dogs $[32,33]$. LA volume measured by RT3DE differed significantly from LA Area, Diameter, LA/AO and M-Mode even after indexation to consider the influence of different body weights.

Table 3 Coefficient of determination (R2) to evaluate the influence of age on left atrial volumes

\begin{tabular}{lllll}
\hline Method & LAMax & LAMin & EF & SV \\
\hline R4AL & 0.0358 & 0.0227 & 0.0138 & 0.0297 \\
R4 & 0.0379 & 0.0247 & 0.0091 & 0.0297 \\
L2 & 0.1534 & 0.1589 & 0.0105 & 0.0876 \\
L4 & 0.2125 & 0.1174 & 0.0048 & 0.2097 \\
Biplane & 0.2134 & 0.1627 & 0.0022 & 0.1664 \\
RTTPE & 0.1793 & 0.1044 & 0.0101 & 0.1580 \\
4D-TomTec & 0.1473 & 0.0361 & 0.0186 & 0.1242 \\
\hline Coeffient of detem & & &
\end{tabular}

Coefficient of determination (R2) to evaluate the influence of age on left atrial (LA) maximum (LAMax) and minimum (LAMin) volume as well as on the ejection fraction (EF) and stroke volume (SV) in 50 healthy cats measured with 7 echocardiographic methods: right parasternal 4 chamber view measured with 2D Area/Length method (R4AL) and 2D monoplane Simpson method of discs (R4), left apical two chamber view (L2) and four chamber view (L4) measured with 2D monoplane Simpson method of discs, L2 and L4 combined to biplane volumetry (Biplane), Real Time Triplane Echocardiography (RTTPE) and $4 \mathrm{D}-\mathrm{TomTec}^{\mathrm{TM}}$ analysing software
Table 4 Coefficient of determination (R2) to evaluate the influence of weight on left atrial volumes

\begin{tabular}{|c|c|c|c|c|}
\hline Method & LAMax & LAMin & $\mathrm{EF}$ & SV \\
\hline R4AL & 0.0350 & 0.1289 & 0.0903 & 0.0010 \\
\hline R4 & 0.0352 & 0.1359 & 0.1123 & 0.0007 \\
\hline L2 & 0.1206 & 0.1321 & 0.0126 & 0.0658 \\
\hline Ł4 & 0.1509 & 0.1275 & 0.0007 & 0.1196 \\
\hline Biplane & 0.1661 & 0.1402 & 0.0044 & 0.1202 \\
\hline RTTPE & 0.0816 & 0.0590 & 0.0024 & 0.0642 \\
\hline 4D-TomTec ${ }^{\mathrm{TM}}$ & 0.0348 & 0.0080 & 0.0120 & 0.0307 \\
\hline
\end{tabular}

Coefficient of determination (R2) to evaluate the influence of weight on left atrial (LA) maximum (LAMax) and minimum (LAMin) volume as well as on the ejection fraction (EF) and stroke volume (SV) in 50 healthy cats measured with 7 echocardiographic methods: right parasternal 4 chamber view measured with 2D Area/Length method (R4AL) and 2D monoplane Simpson method of discs (R4), left apical two chamber view (L2) and four chamber view (L4) measured with 2D monoplane Simpson method of discs, L2 and L4 combined to biplane volumetry (Biplane), Real Time Triplane Echocardiography (RTTPE) and $4 \mathrm{D}-$ TomTec $^{\mathrm{TM}}$ analysing software

In summary, there is a lack of studies using volumetric examinations in feline cardiology. Since these methods are clearly superior to diameter measurements in humans and dogs, they are of high interest for feline medicine as well. The aim of this study was to evaluate 2DE, RTTPE and RT3DE for volumetric measurement of the left atrium in healthy cats and establish a reference interval for further studies on cats with heart disease. Additionally, the influence of age, sex and weight on left atrial volume (LAV) was tested.

\section{Results}

\section{Image acquisition}

Out of 72 cats, 50 animals approved to be suitable for data acquisition. Insufficient $3 \mathrm{DE}$ loop quality was the main reason for dismissing patients. In these 20 cats, the resolution of the $1 \mathrm{DE}$ and $2 \mathrm{DE}$ frames was adequate, but the 3DE data was too blurred to be evaluated. In two patients, the ECG was incomplete and therefore prohibited correct analysis. The results are summarised as mean value \pm standard deviation in Table 1 . Significant differences between the measurement techniques are shown in Table 2.

\section{Influence of age, sex and weight}

The influence of age and weight on the LAV was calculated with the coefficient of determination $R^{2}$. The results for age (range of 0.004-0.25) as well as for weight (range of 0.002-0.17) were below 0.26 with all methods. For LAMax, the influence of weight had even lower $\mathrm{R}^{2}$ values than that of age. The results for weight did not exceed 0.17 (R4AL 0.0350, R4 0.0352, L2 0.1206, L4 0.1509 , Biplane 0.1661, RTTPE 0.0816, 4D-TomTec ${ }^{\text {st }}$ 0.0348). Also with the influence of age, no clear pattern could be distinguished between the methods (R4AL 
0.0358, R4 0.0379, L2 0.1534, L4 0.2125, Biplane 0.2134, RTTPE 0.1793, 4D-TomTec ${ }^{\text {tw }}$ 0.1473). The results are summarized in Tables 3 and 4.

Male LAV results were consistently slightly higher than those of female cats with a range of $+10.46 \%$ to + $19.58 \%$ between the different methods. The overall mean difference of LAV measurements between males and females for LAMax was $0.30 \mathrm{ml}$ with a standard deviation of $0.73 \mathrm{ml}$, and for LAMin it was $0.14 \mathrm{ml}$ with a standard deviation of $0.40 \mathrm{ml}$. The differences were significant in monoplane and biplane methods, but not using three dimensional echocardiography. The results are summarized in Table 5.

\section{Comparison of monoplane SMOD and Area/Length}

R4 was measured with the Simpson as well as the A/L method. They proved to be significantly different $(p<$ 0.05). As a consequence, the two methods cannot be used equivalently. The American Association of Echocardiography recommends SMOD over A/L for LA volumetry in human medicine [12]. Therefore, the Simpson technique was used to calculate the volumes in the other monoplane and biplane views in this study.

\section{LAMax and LAMin}

\section{Monoplane and biplane measurements of LAMax}

R4 had no significant difference to $12(p=0.6690$, bias $=$ $0.032)$ and biplane SMOD $(p=0.7009$, bias $=-0.025)$. Those three methods resulted in significantly higher volumes than RTTPE and 4D-TomTec. L4 showing the lowest monoplane volumes differed from all 2D methods, but not from RTTPE $(p=0.5752$, bias $=$ $-0.0306)$.

\section{D measurements of LAMax}

RTTPE and 4D-TomTec ${ }^{\text {Tix }}$ differed significantly ( $p=$ 0.0135 , bias $=-0.1266$ ). $4 \mathrm{D}-\mathrm{TomTec}^{\mathrm{Tw}}$ quantified the lowest LAMax volumes of all methods. RTTPE results were significant lower than $\mathrm{r} 4 \quad(p=0.0042$, bias $=-0$ :
025), $12(p=<0.0001$, bias $=-0.2442)$ and biplane volumes $(p=<0.0001$, bias $=-0: 1872)$, but had no significant difference to $14(p=0.5752$, bias $=-0.0306)$.

\section{Monoplane and biplane measurements of LA min}

LAMin using $\mathrm{r} 4$ and 12 had no significant difference and $(p=0.3364)$ produced the highest results of all methods. L4 results were significantly lower than all other volumes. Biplane differed significantly from all monoplane measurements with higher results than 14 and lower results than 12 and $\mathrm{r} 4$. No significant difference was found between 12 and 4D-TomTec ${ }^{\text {Tx }}(p=0.0869)$, as well as between R4 and RTTPE $(p=0.0914)$ and 4D-TomTec ${ }^{\mathrm{mm}}$ $(p=0.2473)$.

\section{D measurements of LAMin}

RTTPE and 4D-TomTec ${ }^{\text {TM }}$ resulted in exactly the same mean value $(p=0.9260)$. Furthermore, there was no significant difference between 3D, biplane and 12 volumes. L4 resulted in significantly lower and $\mathrm{r} 4$ in significantly higher volumes than $3 \mathrm{D}$.

\section{$E F$}

Again, $\mathrm{r} 4$ and 12 showed no significant difference $(p=$ 0.5530). The highest EF was measured with 14, which differed significantly from all other methods. Biplane results were significantly different from all other methods as well. 3D measurements did not differ significantly from $\mathrm{r} 4$ and 12 .

\section{SV}

SV values of r4, 12, 14 as well as biplane showed no significant differences with nearly identical mean raw values. RTTPE resulted in significantly lower values than those methods. However, the significantly lowest mean SV was measured with $4 \mathrm{D}-\mathrm{TomTec}^{\mathrm{TN}}$.

Table 5 Comparison of left atrial volumes in healthy male $(n=26)$ and female $(n=24)$ cats

\begin{tabular}{|c|c|c|c|c|c|c|c|c|}
\hline & \multicolumn{4}{|c|}{ LAMax } & \multicolumn{4}{|c|}{ LAMin } \\
\hline & Male & Female & $\mathrm{MD} \mathrm{ml}$ & MD \% & Male & Female & $\mathrm{MD} \mathrm{ml}$ & MD \% \\
\hline R4 & 2.26 & 1.89 & 0.37 & 19.58 & 0.88 & 0.68 & 0.19 & 27.79 \\
\hline L2 & 2.11 & 1.78 & 0.33 & 18.54 & 0.83 & 0.64 & 0.18 & 28.13 \\
\hline$\llcorner 4$ & 2.14 & 1.81 & 0.33 & 18.01 & 0.83 & 0.70 & 0.13 & 18.76 \\
\hline Biplane & 1.90 & 1.63 & 0.28 & 17.11 & 0.59 & 0.48 & 0.12 & 24.23 \\
\hline RTTPE & 2.09 & 1.76 & 0.33 & 18.75 & 0.72 & 0.59 & 0.13 & 22.03 \\
\hline 4D-TomTec ${ }^{\mathrm{TM}}$ & 1.84 & 1.63 & 0.21 & 12.88 & 0.73 & 0.62 & 0.11 & 17.74 \\
\hline
\end{tabular}

Comparison of left atrial maximum and minimum volumes (LAMax/LAMin) in male ( $n=26)$ and female $(n=24)$ healthy cats measured with 7 echocardiographic methods: right parasternal 4 chamber view measured with 2D Area/Length method (R4AL) and 2D monoplane Simpson method of discs (R4), left apical two chamber view (L2) and four chamber view (L4) measured with 2D monoplane Simpson method of discs, L2 and L4 combined to biplane volumetry (Biplane), Real Time Triplane Echocardiography (RTTPE) and 4D-TomTec ${ }^{\mathrm{TM}}$ analysing software. Mean difference (MD) of LAV between male and female was evaluated with a tTest. Significant differences $(p<0.05)$ are marked as bold letters 
Inter- and Intraobserver variability

Inter- and intraobserver variability for LAMax and LAMin was expressed as CV in percent and summarized in Table 6. Interobserver variability was generally higher in LAMin than in LAMax measurements. Biplane (5.59\%) and RTTPE (5.55\%) resulted in the lowest interobserver variability for LAMax, while R4 (20.28\%) had by far the highest values. Interobserver variability was generally high for LAMin and LAMax, ranging between $25.91 \%$ and $47.06 \%$.

\section{Analysis time}

The mean amount of time necessary for completing the various techniques of offline volumetry are summarized in Table 7. Basically, the time rises with the number of dimensions measured. The only exception is the biplane method, which took $10 \mathrm{~s}$ longer than the RTTPE technique. 4D-TomTec ${ }^{\mathrm{Ts}}$ took 8.4 times as long as the simple monoplane diameter measurement and 4.1 times as long as the monoplane Simpson method. R4AL and R4 values were simultaneously calculated because they are based on the same measurements. Therefore their durations are identical. Apart from these two, there was a significant difference between measurement durations of each method $(p=<0.005)$.

\section{Discussion}

The main finding of this study is that it is possible to measure two, three and four dimensional volumes of the left atrium in healthy cats. Apart from that, reference values were established for all methods and one common LAMax reference value could be defined for $12, \mathrm{r} 4$ and biplane SMOD. Bodyweight and age did not have a significant effect on the results, while male cats produced slightly larger values than females.

Table 6 Inter- and intraobserver variability

\begin{tabular}{|c|c|c|c|c|}
\hline & \multicolumn{2}{|c|}{ CV interobserver (\%) } & \multicolumn{2}{|c|}{ CV intraobserver (\%) } \\
\hline & LAMax & LAMin & LAMax & LAMin \\
\hline \multicolumn{5}{|c|}{ Excluding Appendage } \\
\hline R4 & 20.28 & 27.19 & 28.72 & 33.78 \\
\hline L2 & 13.30 & 30.87 & 31.82 & 31.17 \\
\hline L4 & 9.52 & 16.81 & 27.68 & 37.04 \\
\hline Biplane & 5.59 & 18.22 & 25.91 & 32.31 \\
\hline RTTPE & 5.55 & 15.25 & 29.89 & 33.82 \\
\hline 4D-TomTec ${ }^{\mathrm{TM}}$ & 16.63 & 28.93 & 32.30 & 47.06 \\
\hline
\end{tabular}

Inter- and intraobserver variability for left atrial maximum (LAMax) and LA minimum (LAMin) expressed as coefficient of variation (CV) in percent (\%). LAMax and LAMin were measured with 6 echocardiographic methods: 2D monoplane Simpson method of discs (R4), left apical two chamber view (L2) and four chamber view (L4) measured with 2D monoplane Simpson method of discs, L2 and L4 combined to biplane volumetry (Biplane), Real Time Triplane Echocardiography (RTPE) and 4D-TomTec ${ }^{\mathrm{TM}}$ analysing software
Table 7 Comparison of measurement time in seconds

\begin{tabular}{|c|c|c|c|c|}
\hline Analysis Time & Mean & SD & Min & Max \\
\hline R4AL & 20.62 & 2.18 & 17.09 & 24.92 \\
\hline R4 & 20.62 & 2.18 & 17.09 & 24.92 \\
\hline L2 & 22.03 & 1.85 & 18.36 & 28.47 \\
\hline L4 & 23.50 & 2.83 & 18.27 & 29.53 \\
\hline Biplane & 49.67 & 4.04 & 41.72 & 55.76 \\
\hline RTTPE & 39.66 & 1.64 & 37.21 & 43.11 \\
\hline 4D-TomTec ${ }^{\mathrm{TM}}$ & 85.29 & 9.80 & 76.62 & 106.48 \\
\hline
\end{tabular}

Mean, standard deviation (SD), minimum (Min) and maximum (Max) duration in seconds (sec.) for left atrial volumetry in 50 healthy cats measured with 7 echocardiographic methods: right parasternal 4 chamber view measured with 2D Area/Length method (R4AL) and 2D monoplane Simpson method of discs (R4), left apical two chamber view (L2) and four chamber view (L4) measured with 2D monoplane Simpson method of discs, L2 and L4 combined to biplane volumetry (Biplane), Real Time Triplane Echocardiography (RTTPE) and 4DTomTec $^{\mathrm{TM}}$ analysing software. There was a significant difference between measurement durations of each method $(p=<0.005)$

\section{Volumetry}

Echocardiography in awake cats is not easy because of the animal's movement and small LA size. Artifacts due to movement and therefore inadequate quality of $3 \mathrm{DE}$ data were the main reason for excluding patients. Out of 72 cats, 50 animals approved to be suitable for data acquisition. In 20 cats, the resolution of the $1 \mathrm{DE}$ and $2 \mathrm{DE}$ frames was adequate, but the $3 \mathrm{DE}$ data was too blurred to be evaluated. In two patients, the ECG was incomplete and therefore prohibited correct analysis. Furthermore, the small size of a cat's atrium requires very high image resolution. The high heart rate makes a high frame rate necessary to ensure adequate temporal resolution.

\section{Monoplane methods}

Monoplane r4, 12 and biplane resulted in mean values that are equal to the first decimal place and did not show statistic difference for LAMax $(p>0.05)$. Therefore they can be used equivalently for LAMax evaluation in future studies. One reference range for all three methods was set up: $1.96 \pm 0.54 \mathrm{ml}$ (Table 1). Those three methods produced significantly higher volumes than RTTPE and 4D-TomTec ${ }^{\mathrm{Tm}}$. This accords with former findings in human medicine, where monoplane $2 \mathrm{D}$ methods measured higher LAV than 3DE due to geometric assumptions instead of endocardial border detection $[12,34,35]$. R4 monoplane SMOD values in a comparative study were higher $\left(2.24 \mathrm{~cm}^{3}\right.$ mean value) than those in this study $\left(1.95 \mathrm{~cm}^{3}\right.$ mean value $)$. However, only 21 cats were included and only 11 were European Short Hair [5]. L4 had lower results and therefore differed from all 2D methods except for RTTPE ( $p=$ 0.5752 ). The significant difference between 12 and 14 LA volumes is due to the ovoid LA shape and therefore different diameters. This can be seen well in 3D-images, 

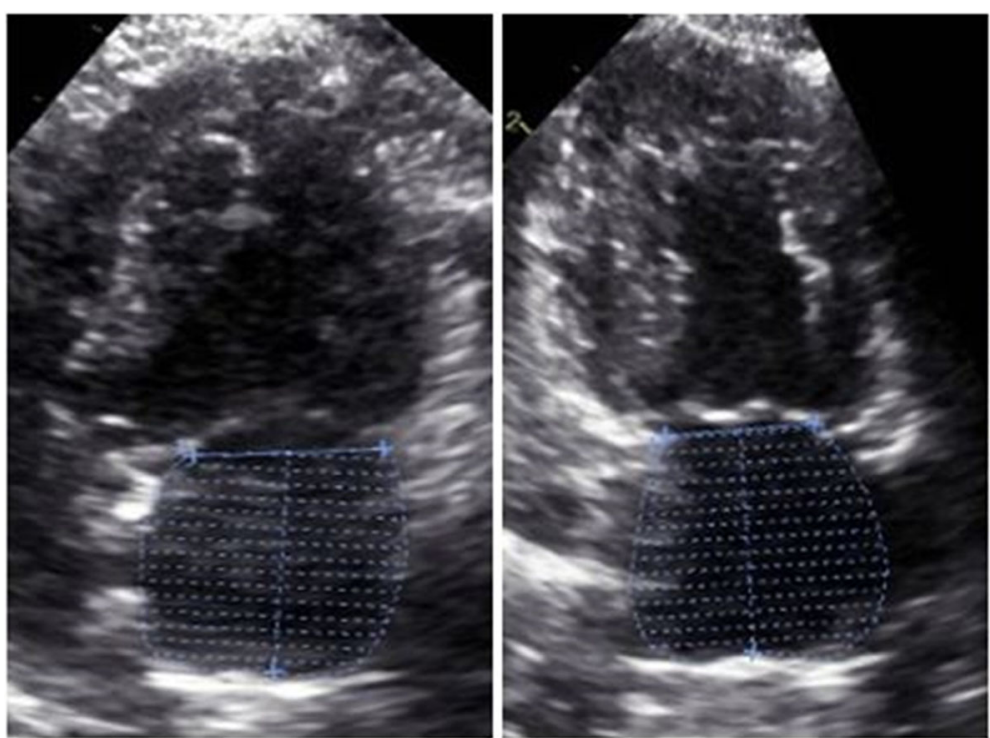

Fig. 1 Left atrium (LA) volume measurement using monoplane Simpson method of discs (SMOD): Representative images of left atrial maximum volume (LAMax) measurement in a healthy cat with SMOD in left apical 4 chamber view (left) and left apical 2 chamber view (right)

where both planes are displayed concurrently (Fig. 2, Fig. 3). No veterinary study exists on the comparison of 12 and 14 . We expected $\mathrm{r} 4$ and 14 to be similar, as LVvolumes in dogs using these planes were the same [29]. However, there was a small $(0.2 \mathrm{ml})$ but significant $(p=$ $0.126)$ difference. This could be caused by a slightly oblique angle and the ovoid shape of the LA. Because of the flattened position of the feline heart, an optimal long axis view is difficult to achieve in some cats. A similar effect is described in older humans caused by alterations of the position of the diaphragm [12]. Based on our results, two limit values are necessary for monoplane LA. This finding is shared in a comparable new study in cats, where the mean values for $\mathrm{r} 4(1.95 \mathrm{ml})$ and $14(1.77 \mathrm{ml})$ LA SMOD are exactly the same as ours [26].

Also for LAMin, results from the 14 plane were significantly lower than all other monoplane volumes and R4 and 12 did not differ significantly $(p=0.3364)$. In a comparative study with higher monoplane SMOD LAMax values, the LAMin values are higher too (range of 0.73 to $1.22 \mathrm{ml}$ ). These consistently higher results justify the assumption that measurements were performed more generously than in this paper and inter-observer variability mismatches [5]. In a new comparable study with 162 cats, the mean monoplane SMOD measurements in the 14. plane equal ours to the first decimal space and in the
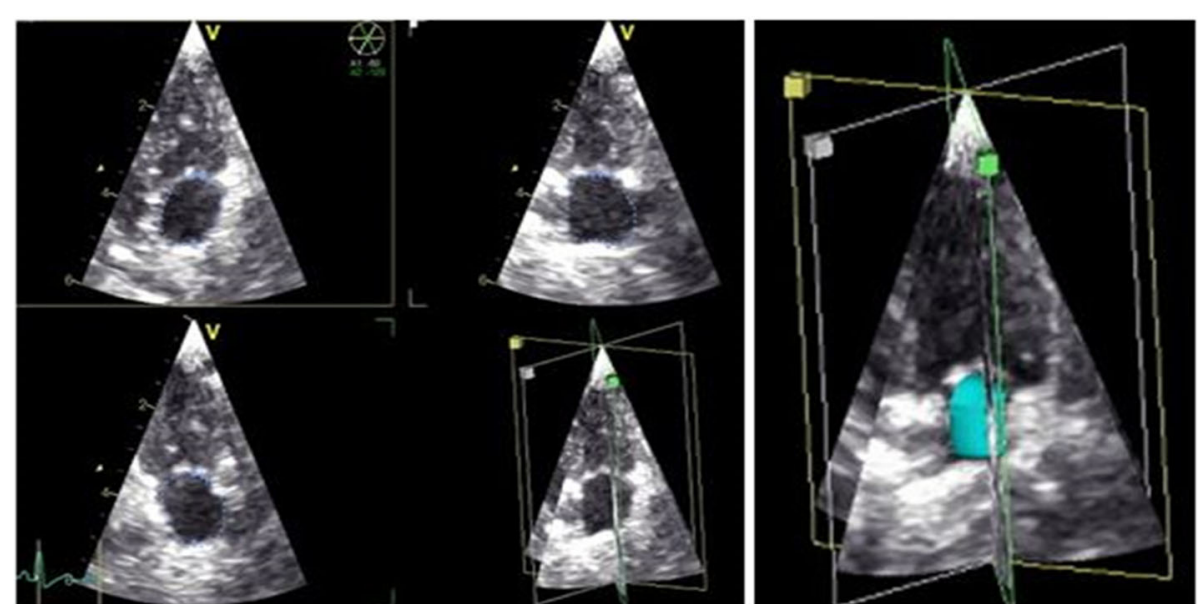

Fig. 2 Left atrium (LA) volume measurement using Real Time Triplane Echocardiography (RTTPE): Representative images of left atrial maximum (LAMax) volumetry in a healthy cat with RTTPE (left). The atrium is concurrently displayed in all three angles. The analysis result is shown as three dimensional dynamic reconstruction of LA (right) 


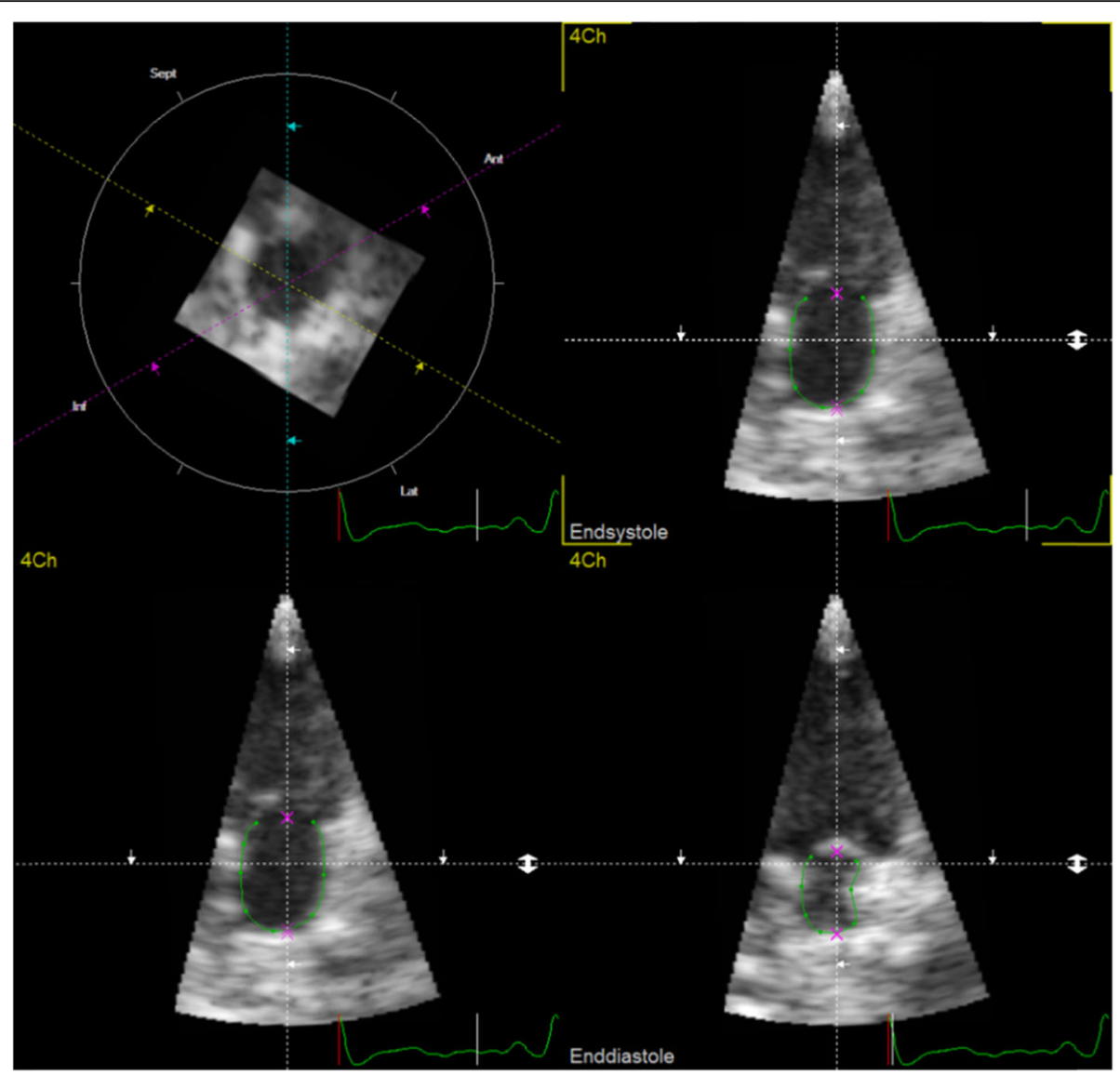

Fig. 3 Left atrium (LA) volume measurement using Real Time 3 dimensional Echocardiography (RT3DE) with 4DE-TomTec ${ }^{\mathrm{TM}}$ as evaluation program: Representative images of LA volumetry in a healthy cat with RT3DE. The atrium is concurrently displayed in dynamic short axis view, left apical 4 chamber view, left apical 2 chamber view and left apical 3 chamber view

r4 plane they only differ by $0.1 \mathrm{ml}$. It was suggested that LAMin was superior to LAMax to distinguish cardiomyopathy and congestive heart failure [26]. As LAMin results are very low ( 0.5 to $0.8 \mathrm{ml})$, the parameter appears to be prone-to-error. Therefore we do not concur and do not recommend LAMin over LAMax.

EF and SV have large standard deviations (SD) in all methods. This can be explained by the small volumes, where minor variation has major impact. In an already mentioned study on monoplane LA volumetry in cats, EF had similarly large SD. It generally was decreased in cats with cardiac pathologies in comparison to healthy ones, but could not distinct decompensated from asymptomatic HCM [5]. EF therefore seems unsuitable as sole indicator for LA enlargement in cats. In dogs it was also proven, that EF tends to vary widely between animals and measurement techniques and is discouraged as independent marker for the left atrium [32]. In humans, LA has greater volume $(22-52 \mathrm{ml})$ and results are advised to be indexed to body surface area, which leaves EF and SV without large SD [12].

\section{Biplane method}

Since biplane SMOD did not show significant difference to LAMax results of monoplane $\mathrm{r} 4$ and 12 , measuring two planes does not provide additional benefit in healthy cats. In human medicine, no significant difference between monoplane and biplane SMOD was found in healthy patients as well as patients with cardiac diseases [36]. Nevertheless, the American Association of Echocardiography and the European Association of Cardiovascular Imaging recommend biplane SMOD as standard technique for LA volumetry, because monoplane SMOD formula assumes circular LA shape in the short axis view, which is not always accurate [12]. The absent advantage of biplane over monoplane SMOD in healthy cats may be explained by the very small volumes. Mean values of 14 and 12 differ significantly, but by only $0.2 \mathrm{ml}$ and are obviously relativized in the SMOD formula. Additionally, biplane SMOD requires significantly more time for retrograde measurement than monoplane measurement. In summary, monoplane SMOD appears to be the preferable alternative to biplane SMOD for 2D LA volumetry in healthy cats. . 

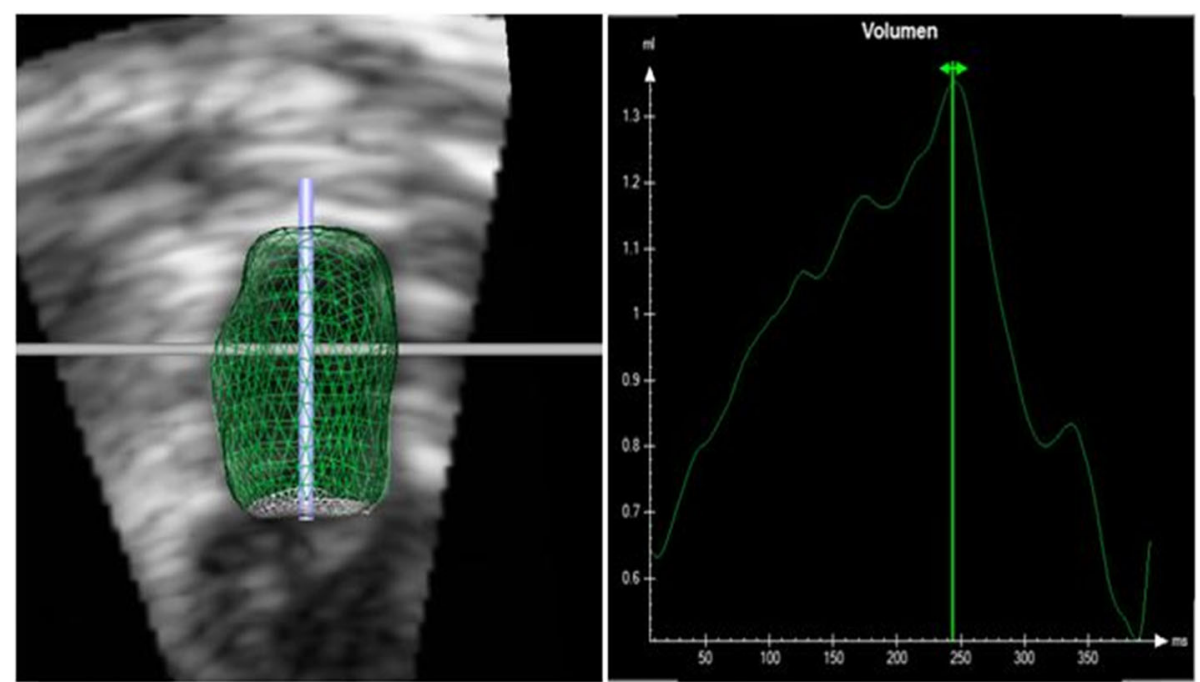

Fig. 4 Left atrium (LA) volume measurement using Real Time 3 dimensional Echocardiography (RT3DE) and 4DE-TomTec ${ }^{T M}$ as evaluation program: Representative RT3DE analysis of the left atrium in a healthy cat. The dynamic 3D model of LA as well as the time volume curve is displayed

\section{Triplane echocardiography}

LAMax results were significantly lower than those of monoplane ( $\mathrm{r} 4$ and 12 ) and biplane SMOD. These findings are contrary to LV volumetry in dogs, where RTTPE values were significantly higher than monoplane SMOD and identical with CMRI [29]. However, LA and LV have a different shape, which makes results and relations not comparable. In cats, no comparison of RTTPE and cMRI has been done yet. Therefore, it also has to be considered, that maybe 12 and $\mathrm{r} 4$ are false high and RTTPE is not false low. This could be explained by the longitudinal-oval LA shape. The automatically generated discs are circular since the method was originally designed for LV volumetry. Circular discs with 12 diameter would project beyond the outer rim of the oval atrium in every layer and produce false high results. RTTPE consequently may capture one plane with high volume (12) and two planes with significantly lower volume (13, 14).

LAMin was difficult to measure especially with 3D methods, due to limited image quality and barely options for adaption during analysis, caused by the small size of the normal feline left atrium. The manual border detection was more difficult than at monoplane measurements due to the low frequency probe. RTTPE is inferior to 2DE, but superior to RT3DE in terms of spatial resolution. Out of the 20 cats that were excluded from measurements due to too low image quality, only 4 were dismissed because of problems with RTTPE loops. Frame rate was above $40 \mathrm{fps}$ in both 3DE methods, but above 60 in $2 \mathrm{DE}$. The necessity of a special probe has to be rated negatively in terms of costs and practicability caused by the great size and weight. An advantage of RTTPE is the fast retrograde measurement. It only took twice as long as monoplane and even $10 \mathrm{~s}$ less than biplane SMOD. From all these reasons, it can be assumed, that RTTPE works well for LAV in healthy cats and using three planes perhaps provides more realistic results than mono- or biplane SMOD, but further studies are required for verification by comparison with cMRI.

\section{Real time three dimensional echocardiography}

RT3DE is supposed to be the most realistic and best echocardiographic method to capture the true LAV. In human LA volumetry, RT3DE has excellent correlation with cMRI and usually larger volumes than $2 \mathrm{DE}[12,37$, 38]. In our study, 4D-TomTec ${ }^{\mathrm{Tu}}$ quantified the lowest LAMax volumes of all methods. This is also the case in LA and LV volumetry in dogs $[29,33]$. One possible explanation is the low image resolution due the low frequency probe. This decreased the quality of the automatic endocardial border detection and impeded the manual corrections, since the agitated endocardial border seemed broadened. This also led to the longest measurement-duration of all compared methods. An additional problem was the temporal resolution which could have caused deviating results too. 3DE achieved frame rates of approximately 40-50 fps, which equals 12 to 20 images per heartbeat at a cat's heartrate of 120 to 240 per minute. In human medicine a frame rate of 30 is reached for a heartrate of 60 to 100 beats per minute [39]. The Vivid $\mathrm{E} 9^{1}$ is able to create a volume block by using multiple subvolumes of ECG triggered consecutive $\mathrm{R}-\mathrm{R}$ cycles, to attain higher frame rates and therefore better resolution. However, because of the small amplitude of cat's ECGs, the software had problems

\footnotetext{
${ }^{1}$ Vivid E9, GE Healthcare, Horten, Norway.
} 

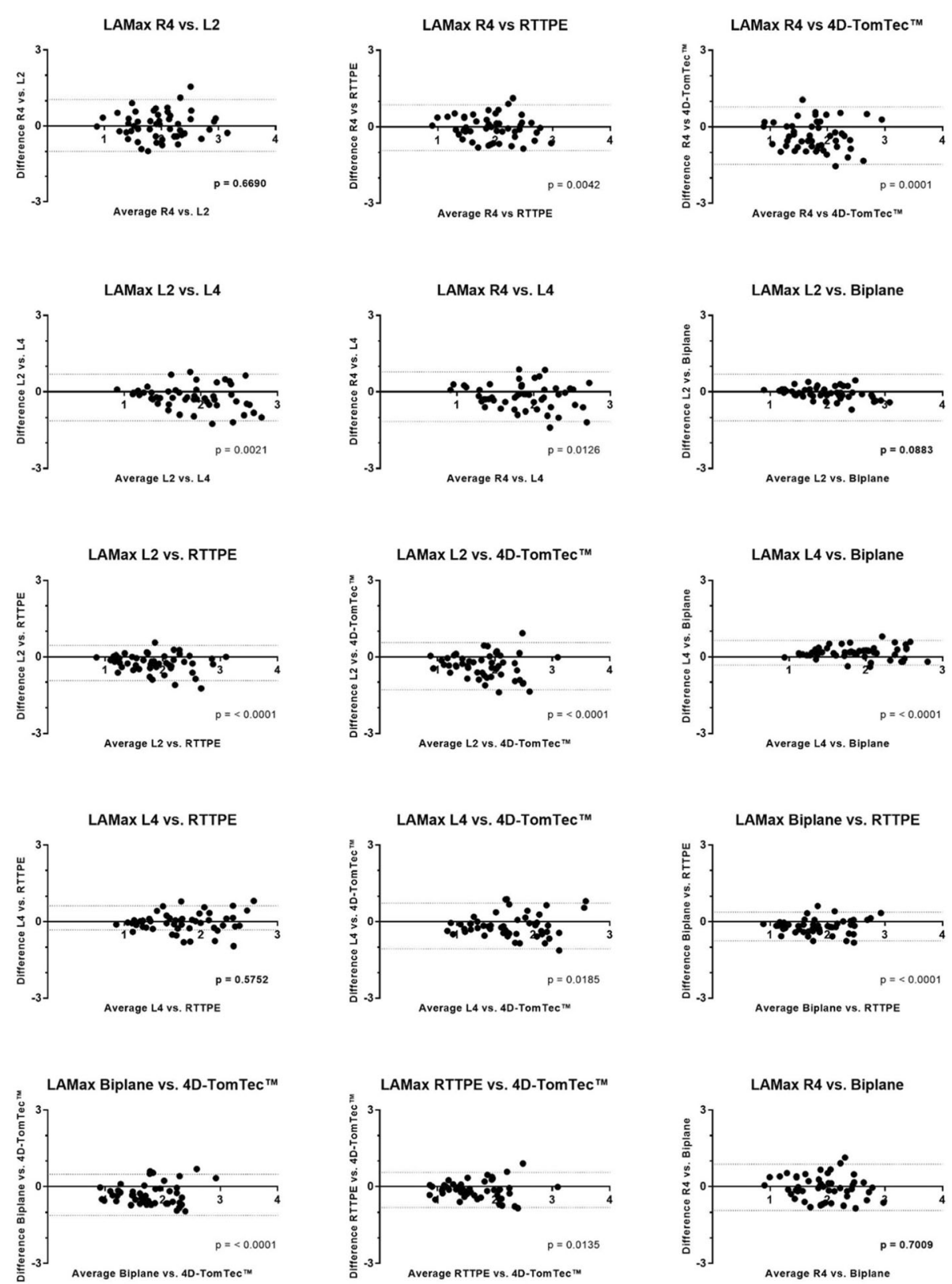

Fig. 5 Bland-Altman plots comparing 6 different methods for left atrial maximum volume (LAMax) quantification: The methods are: monoplane Simpson method of discs (R4), left apical two chamber view (L2) and four chamber view (L4) measured with 2D monoplane Simpson method of discS, L2 and L4 combined to biplane volumetry (Biplane), Real Time Triplane Echocardiography (RTTPE), 4D-TomTec ${ }^{\mathrm{TM}}$ analysing software. Mean differences between the measurement-techniques were evaluated with a t-Test. P $>0.05$ was evaluated as significant similarity and marked as bold letters

recognising the R-peak and produced irregular R-R distances. A reliable fusion of subvolumes could therefore not be performed.

Maybe the evaluation of LA with 3DE is less problematic in cats with cardiac pathologies and dilated atria. This will have to be verified in further studies. Currently, 3DE is limited for LA evaluation in cats due to the tiny LA size, high heart rates, the demand for a special probe and analysis software, time consuming retrograde measurement and suboptimal image resolution.

Influence of age, sex and weight

The coefficient of determination $\mathrm{R}^{2}$ was below 0.25 at all times for all methods when calculated for weight as well as for age. We concluded that LAV is not dependent on age and weight and raw data was used instead of correlated values. The finding concurs with data from human 
medicine. In a healthy human, LA size does not alter until the age of 80 [40]. The influence of age was never tested in cats before, but the effect of weight on LA has earlier been described as significant [15, 41-43]. However, these results were calculated on the base of $\mathrm{M}$ Mode or LAD data and are therefore not comparable with our volumetry results.

In Human Medicine, it is recommend to index results to body surface area [12]. The influence of body surface area on LAV is also proven in dogs [44], but also allometric scaling to the bodyweight was justified [45-47].

In our study, the population almost exclusively consists of European Shorthair cats and the covered weight range is very narrow $(4.42 \pm 1.41 \mathrm{~kg})$ which could explain the lack of influence over LAV. Maybe results would have been different if the tested population would have included more animals from large breeds (e.g. Maine Coons).

Male LAV results were slightly higher than those of female cats with a range of $+10.46 \%$ to $+19.58 \%$. The differences were significant in monoplane and biplane methods. These results go well with former findings in cats and humans [12, 15, 41]. Nevertheless, a cat's LAMax is $1.95 \pm 0.56 \mathrm{ml}$ with monoplane SMOD (r4), so a plus of $20 \%$ would only mean a difference of approximately $0.2 \mathrm{ml}$. Under reservation, gender specific reference values were established, but further studies in cats with LA Dilatation are necessary to evaluate, if gender specific limit values are really required.

\section{Inter- and Intraobserver variability}

Generally, CV is remarkably high. This is probably due to the very low mean value and the high standard deviation in all methods. In a paper evaluating LA volumes in 40 dogs, CV was similarly high because of the same reason [48]. No clear distinction can be drawn between $\mathrm{CV}$ of either left apical and right parasternal views or the different measurement methods. Further studies in cats with cardiac pathologies are necessary to evaluate if inter- and intraobserver variability improves with pathologically enlarged LA volumes.

\section{Limitations}

LAV was not measured with the golden standard cMRI. Therefore it is not possible to determine which echocardiographic method is the most realistic and reliable method. On the other hand it was never the aim of the study to elect the best method, but to evaluate which methods are possible, similar in their results and suitable for everyday use. Secondly, LA volumes in healthy cats are very small. There is a chance, that measurements are slightly more imprecise and more susceptible to minor variations than in LV measurements. Possibly, volumetry in cats with cardiac pathologies and dilated LA is easier and faster to perform. This of course requires confirmation by further studies. Also, we did not test the cats for hyperthyroidism. The disease primarily affects LV measurements and all cats were free of medical history, but nevertheless it would have rounded off the anamnesis if we had tested the blood value. Finally, the study is limited due to the small number of examined cats. To set up reference values by confidence intervals, it is advised to include at least 120 animals [49]. We included only 50 cats, but used mean $\pm \mathrm{SD}$, which is the recommended way to present truthful values from group of 40 to 120 participants [50].

\section{Conclusion}

This study shows that monoplane, biplane, RTTPE and 4D-TomTec ${ }^{\text {rx }}$ are applicable methods to assess left atrial volume in healthy cats. Most results differentiate significantly and therefore cannot be directly compared or exchanged in future studies. Only monoplane $12, \mathrm{r} 4$ and biplane SMOD do not differ significantly and can be understood as equivalent. Furthermore, raw volume data can be used and does not need to be correlated with the cat's weight or age. Male cats have significantly larger atria than females, therefore the set-up of sex related limit values for LA enlargement seemed legitimate.

\section{Methods}

This study was approved by the Ethical Committee of the University of Veterinary Medicine Hannover and not classified as animal experiment as data was collected during regular cardiac examination.

\section{Animals}

For this study, 50 unsedated cats underwent echocardiographic examination (26 male, 24 female, $6.48 \pm$ 3.05 years, 48 European Shorthair, 1 Maine Coon and 1 Burmese, median weight of $4.42 \pm 1.41 \mathrm{~kg}$ ). The animals were patients of the Small Animal Hospital of the University of Veterinary Medicine Hannover. First, all 50 cats underwent clinical examination and blood pressure measurement. Then, the echocardiographic examination was performed. Following inclusion requirements had to be met: 1 . The anamnesis was without history of cardiac pathologies, hypertonia, syncope and dyspnoea, 2. The clinical examination was clear of pathological findings, 3. The systolic blood pressure did not exceed $160 \mathrm{mmHg}$, 4. The echocardiography and ECG did not show any abnormalities, 5. The anteroposterior diameter of the left atrium, measured in the right parasternal long axis, was below $1.6 \mathrm{~cm}$, and 6 . The LA/AO ratio was below 1.4 in the right parasternal short axis view. 


\section{Echocardiography}

All echocardiographic exams were performed by one experienced veterinarian (S.O.H.) with a commercially available ultrasound unit ${ }^{1}$. It was equipped with a $12 \mathrm{~S}-\mathrm{D}$ Phased Array probe for 1DE and 2DE ultrasonography (4-12 MHz) and a $3 \mathrm{~V}$ Matrix Array probe for 3D examination $(2.5-3.6 \mathrm{MHz})$. The cats were laid in right lateral recumbency for the right parasternal long and short axis view and in left lateral position for left apical 2, 3 and 4 chamber view. The recommendations for cardiac chamber quantification by echocardiography of the American Society of Echocardiography were followed as closely as possible in the performance of the different techniques and are described in the chapters below [12]. Also, the ultrasound units' ECG was attached and displayed during the examinations. For every plane, loops of at least three consecutive heart beats were recorded and saved for offline analysis. All data was transferred to a workstation $^{2}$ for offline analysis.

\section{Measurement of left atrial diameter and $L A / A O$}

LAD measurement was performed in the same way the reference value of $1.6 \mathrm{~cm}$ was described $[8,11]$. Both atria, both ventricles and the mitral and tricuspid valve were completely displayed. LAD was measured in ventricular end systole, defined as the moment exactly before mitral valve opening (LAMax) [9].

$\mathrm{LA} / \mathrm{AO}$ measurement was performed in the right parasternal short axis view with the 'Swedish method' as described in dogs [51]. A limit value of 1.4 was used to classify healthy animals $[1,14]$. The inner-edge to inner edge measurement of LA and Aortic valve was performed in early ventricular diastole, defined as the frame directly after closure of the aortic valve.

\section{Left atrial volumetry}

Left atrial maximum volume (LAMax) was measured directly before opening of the mitral valve, in ventricular end systole, one or two frames after the ECG's T-wave. Left atrial minimum volume (LAMin) was measured directly after closing of the mitral valve at the end of LV diastole, one or two frames after the ECG's P-wave. LAMax and LAMin, ejection fraction (EF) and stroke volume (SV) were measured with monoplane 12,14 and r4, as well as biplane, RTTPE and 4D-TomTec ${ }^{\text {trit. }}$.

\section{Monoplane examination}

For two-dimensional monoplane LA volumetry, the right parasternal 4 chamber view (r4), as well as the left apical 2 (12) and 4 (14) chamber views were displayed (Fig. 1) [12]. Attention was paid to avoid foreshortening by

${ }^{2}$ EchoPAC PC, 108.1.4 Version 110.x.x, GE Healthcare, Horten Norway. depicting the maximum (LV) length, the whole LA and in the 12 plane also the complete LAA. After LAMax and LAMin were assessed with Area/Length (A/L) for $\mathrm{r} 4$ and SMOD for $\mathrm{r} 4,14$ and 12 , the program automatically calculated EDV, ESV, ejection fraction (EF) and stroke volume (SV). SV describes the output during one systole in millilitres (ml). EF is the percentage of blood volume that is ejected by the left atrium during one heartbeat.

\section{Biplane examination}

For biplane examination the corresponding 12 and 14 planes were combined according to the recommendations of the American Society of Echocardiography [12].

\section{Real time triplane echocardiography (RTTPE)}

The RTTPE uses three planes, which are in an angle of $60^{\circ}$ to each other. Based on the measurements in each of these planes, the software calculates a dynamic 3D reconstruction of the LA volume during the measured heart phase. This method was performed in the left apical axis of the heart. First, 14 view without signs of foreshortening was adjusted and then corrected until the concurrently displayed 12 and 13 planes depicted the entire LA including LAA. The width and depth of the frame was adjusted for closest capture of the left atrium to achieve frame rates above 40 frames per second (fps). The data sets were transferred to the workstation and measured later on. The definition of the time points for measurement were the same as described for the $2 \mathrm{DE}$ methods. At the beginning of offline analysis, the moment of LAMax was adjusted. Then, LA was traced along the endocardial border from the septal to the parietal mitral valve annulus in all three planes. The contour was automatically closed by connecting the opposite segments at mitral valve level. Attention was paid to exclude the pulmonary trunk as well as LAA. Next, the moment of LAMin was selected and the three planes were traced in the same pattern. Derived from these 6 measurements, the program automatically generated a dynamic reconstruction of the LA for the current heart cycle (Fig. 2). Additionally, LAMax, LAMin, EF and SV were calculated.

\section{Real-time three dimensional echocardiography with border detection}

RT3DE was performed in the left apical axis as described for RTTPE examinations [12]. The Vivid E9 is able to create a volume block by using multiple subvolumes of ECG triggered consecutive $\mathrm{R}-\mathrm{R}$ cycles, to attain higher frame rates and therefore better spatial resolution. However, because of the small size of the feline heart, frame rates above $40 \mathrm{fps}$ were reached by using just one sub volume. Therefore, we truly achieved a real time $3 \mathrm{D}$ examination. LAV quantifications were offline 
performed with the $4 \mathrm{D}$-TomTec ${ }^{\mathrm{ma}}$ program. ${ }^{3}$ The first step was to centre LA manually in all planes to avoid foreshortening. The program was designed to quantify ventricles, so end-diastole and end-systole of the ventricle were detected automatically based on the ECG. In contrast to the RTTPE method, 4D-TomTec ${ }^{\text {rm }}$ displayed one axis at a time with both, end-systole and enddiastole. The atrial endocardial border was detected automatically and had to be corrected manually for optimal tracing. It was made sure to exclude pulmonary veins as well as the left appendage. After tracing the endocardial border in these six pictures, a border detection of the whole heartbeat started. Manual adaption of the border lines in all frames of this heart beat were performed, in order to achieve the best alignment (Fig. 3). Finally, LA was reconstructed as a dynamic 3D body and a volume curve for the edited heart cycle was calculated (Fig. 4). Based on this volume curve, maximum and minimum volume were determined and $\mathrm{SV}$ and $\mathrm{EF}$ calculated.

\section{Time for measurements}

The duration of each method was recorded with a stopwatch from the point of opening the data set for analysis until LAMin, LAMax, EF and SV were calculated.

\section{Statistical analysis}

Statistics and graphs were calculated with commercially available software ${ }^{4,5}$ All measurements were performed three times and averaged for statistical analysis. Normal distribution of LAMax, LAMin, EF and SV was verified with the Shapiro-Wilk method. The averaged values were expressed as mean \pm standard deviation (SD) and as $95 \%$ prediction interval in $\mathrm{ml}$. Bland-Altman graphs were created to visualize the relations between different measurement methods for LAMax (Fig. 5). Bias and Limits of Agreement (LOA) are summarized in Table 8. Since $\mathrm{r} 4,12$ and biplane SMOD did not result in significantly different LAMax values, one reference range for the three methods was set up. All LAMax measurement data obtained by these three methods was used to calculate a common Mean and SD. A descriptive statistical analysis was used for age, body weight and sex. The influence of age and weight on the left atrial volume was calculated with the coefficient of determination $R^{2} \cdot R^{2}=$ 1 is regarded as proof for perfect linear correlation, whereas $R^{2}=0$ stands for no linear connection. To detect whether there are LA volume differences in male

\footnotetext{
${ }^{3} 4 \mathrm{D}$ LV-Function ${ }^{\text {nx }}$ 2.2, TomTec Imaging Systems GmbH, Unterschleißheim.

${ }^{4}$ SAS version 9.3, SAS Institute, Cary, NC, USA.

${ }^{5}$ GraphPad Prism, Version 5.00 forWindows, GraphPad Software, San Diego, Calif., USA. 4D-AutoLVQ ${ }^{\mathrm{m}}$, EchoPAC PC, GE Healthcare, Horten, Norway.
}

Table 8 Overview of Bland-Altman analysis results

\begin{tabular}{|c|c|c|c|}
\hline Methods compared & Bias & upper LOA & lower LOA \\
\hline R4 vs L2 & 0.0320 & 1.063 & -0.9989 \\
\hline R4 vs L4 & -0.1816 & 0.7899 & -1.153 \\
\hline R4 vs Biplane & -0.025 & 0.8717 & -0.9217 \\
\hline R4 vs RTTPE & -0.025 & 0.8717 & -0.9217 \\
\hline R4 vs 4 DTomTec ${ }^{\text {TM }}$ & -0.3388 & 0.7891 & -1.467 \\
\hline L2 vs L4 & -0.2136 & 0.6981 & -1.125 \\
\hline L2 vs Biplane & -0.057 & 0.3977 & -0.5117 \\
\hline L2 vs RTTPE & -0.2442 & 0.4469 & -0.9353 \\
\hline L2 vs 4DTomTec ${ }^{\mathrm{TM}}$ & -0.3708 & 0.551 & -1.293 \\
\hline L4 vs Biplane & 0.1566 & 0.6319 & -0.3187 \\
\hline L4 vs RTTPE & -0.0306 & 0.7212 & -0.7824 \\
\hline L4 vs 4DTomTec ${ }^{\mathrm{TM}}$ & -0.1572 & 0.7369 & -1.051 \\
\hline Biplane vs RTTPE & -0.1872 & 0.3797 & -0.7541 \\
\hline Biplane vs 4DTomTec ${ }^{T M}$ & -0.3138 & 0.4862 & -1.114 \\
\hline RTTPE vs 4DTomTec ${ }^{\text {TM }}$ & -0.1266 & 0.5576 & -0.8108 \\
\hline
\end{tabular}

Results of Bland-Altman comparison between LAMax results of 50 healthy cats measured with 6 echocardiographic methods: right parasternal 4 chamber view measured with 2D monoplane Simpson method of discs (R4), left apical two chamber view (L2) and four chamber view (L4) measured with 2D monoplane Simpson method of discs, L2 and L4 combined to biplane volumetry (Biplane), Real Time Triplane Echocardiography (RTTPE) and 4DTomTec $^{\mathrm{TM}}$ analysing software. $(\mathrm{LOA}=$ limit of agreement)

and female cats, simple T-test was calculated. The difference was considered significant when $p<0.05$. For group-comparison single factor variance analyses as well as a paired T-test for multiple pairwise comparisons of the normal distributed data were performed. A $p$-value of less than 0.05 was considered significant. The duration of measurement for each method was normally distributed and therefore specified with mean \pm SD, Min and Max. The increase of volume from female to male left atrial volume (LAV) was calculated with the following formula: LAV Difference $(\%)=\left(\frac{\text { Mean Difference }}{\text { LAV Female }}\right) * 100$. Inter- and intraobserver variability was evaluated by coefficient of variation $(\mathrm{CV})$ for all methods. Intraobserver $\mathrm{CV}$ was calculated as mean divided by standard deviation and expressed as percentage. For interobserver variability, five patients were independently measured by two different observers. $\mathrm{CV}$ was then calculated as standard deviation of the mean difference between the two data sets divided by the total mean and multiplied by 100 .

\section{Abbreviations}

1DE: One dimensional echocardiography; 2DE: Two dimensional echocardiography; 3DE: Three dimensional echocardiography; CMRI: Cardiac MRI; ECG: Electrocardiogram; EDV: End-diastolic volume; EF: Ejection fraction; ESV : End-systolic volume; L2: Left apical two chamber view; L4: Left apical four chamber view; LA : Left atrium; LAA: Left atrial appendage; LAV: Left atrial volume; LA/AO: Proportion of the left atrial diameter to the aortic valve; LAAV: Left atrial appendage volume; LAMax: Left atrial maximum volume; LAMin: Left atrial minimum volume; LV: Left ventricle; MHZ: Megahertz; 
Ml: Mililiter; MM: Milimeter; MMode: Motion Mode; R4: Right parasternal four chamber view; R4AL: Right parasternal four chamber view calculated with Area/Length formula; RT3DE: Real Time 3D Echocardiography; RTTPE: Realtime triplane echocardiography; SD: Standard deviation; SMOD: Simpson method of discs

\section{Acknowledgements}

J.R. is grateful for the support of her colleagues Anna Pilgram, Katja Rumstedt and Davina Ludwig.

\section{Authors' contributions}

J.R., M.F., M.B. and S.O.H designed the study. S.O.H. performed the echocardiographic examinations. J.R. reviewed scientific literature, analysed the data, wrote the manuscript and created the tables and Figs. M.B. calculated the statistics and assisted with the interpretation. M.F. and S.O.H helped with the creation of the manuscript. All authors read and approved the final manuscript.

\section{Funding}

Not applicable.

\section{Availability of data and materials}

The datasets used and analysed during the current study are available from the corresponding author on reasonable request.

\section{Ethics approval and consent to participate}

All patients were privately owned and presented for cardiac examination at the Small Animal Hospital of the University of Veterinary Medicine Hannover. The Ethical Committee of the University of Veterinary Medicine Hannover therefore approved this study and did not classify it as animal experiment.

\section{Consent for publication}

Not applicable.

\section{Competing interests}

The authors declare that they have no competing interests.

\section{Author details}

${ }^{1}$ Small Animal Clinic, University of Veterinary Medicine Hannover, Foundation, Bünteweg 9, D-30559 Hannover, Germany. 'Department of Biometry, Epidemiology and Information Processing, University of Veterinary Medicine Hannover, Foundation, Bünteweg 2, D-30559 Hannover, Germany. ${ }^{3}$ Tiergesundheitszentrum Hungerbühler, Tierärztliche Klinik für Kleintiere Salzgitter, Gerichtsweg 3, 38229 Salzgitter, Germany.

Received: 11 February 2019 Accepted: 13 July 2020

Published online: 29 July 2020

\section{References}

1. Payne JR, Borgeat K, Connolly DJ, Boswood A, Dennis S, Wagner T, et al. Prognostic indicators in cats with hypertrophic cardiomyopathy. J Vet Intern Med. 2013;27:1427

2. Chetboul V, Passavin P, Vassiliki ET, Poissonnier C, Loic JP. Clinical epidemiological and echocardiographic features and prognostic factors in cats with restrictive cardiomyopathy : A retrospective study of 92 cases ( 2001-2015 ). J Vet Intern Med. 2019;33(February 2018):1222-31.

3. Locatelli C, Pradelli D, Campo G, Spalla I, Savarese A, Brambilla PG, et al. Survival and prognostic factors in cats with restrictive cardiomyopathy: a review of 90 cases. J Feline Med Surg. 2018;20(12):1138-43.

4. Losi M-A, Betocchi S, Barbati G, Parisi V, Tocchetti C-G, Pastore F, et al. Prognostic significance of left atrial volume dilatation in patients with hypertrophic cardiomyopathy. J Am Soc Echocardiogr [Internet]. 2009;22(1): 76-81. Available from:. https://doi.org/10.1016/j.echo.2008.11.001.

5. Linney CJ, Dukes-McEwan J, Stephenson HM, López-Alvarez J, Fonfara S. Left atrial size, atrial function and left ventricular diastolic function in cats with hypertrophic cardiomyopathy. J Small Anim Pract [Internet]. 2014;55(4): 198-206 Available from: http://doi.wiley.com/10.1111/jsap.12186.

6. Peterson EN, Moise NS, Brown CA, Erb HN, Slater MR. Heterogenity of hypertrophy in feline hypertrophic heart disease. J Vet Intern Med. 1993;7: 183-9.
7. Fox PR, Keene BW, Lamb K, Schober KA, Chetboul V, Luis V, et al. International collaborative study to assess cardiovascular risk and evaluate long-term health in cats with preclinical hypertrophic cardiomyopathy and apparently healthy cats : the REVEAL study. J Vet Intern Med. 2018;(August 2017):930-43.

8. Schober KE, Maerz I. Assessment of left atrial appendage flow velocity and its relation to spontaneous echocardiographic contrast in 89 cats with myocardial disease. J Vet Intern Med. 2006;20(1):120-30.

9. Schober KE, Maerz I, Ludewig E, Stern J. a. Diagnostic accuracy of electrocardiography and thoracic radiography in the assessment of left atria size in cats: comparison with transthoracic 2-dimensional echocardiography. J Vet Intern Med. 2007;21(4):709-18.

10. Vezzosi T, Schober KE. Doppler-derived echocardiographic evidence of pulmonary hypertension in cats with left-sided congestive heart failure. J Vet Cardiol. 2019;23(September 2017):58-68. Available from. https://doi.org/ 10.1016/j.jvc.2019.01.007.

11. Schober KE, Wetli E, Drost WT. Radiographic and echocardiographic assessment of left atrial size in 100 cats with acute left-sided congestive heart failure. Vet Radiol Ultrasound [Internet]. 2014;55(4):359-67 Available from: http://doi.wiley.com/10.1111/vru.12131.

12. Lang RM, Badano LP, Mor-avi V, Afilalo J, Armstrong A, Ernande L, et al. Recommendations for Cardiac Chamber Quantification by Echocardiography in Adults : An Update from the American Society of Echocardiography and the European Association of Cardiovascular Imaging. J Am Soc Echocardiogr [Internet]. 2015;28(1):1-39.e14. Available from:. https://doi.org/10.1016/j.echo.2014.10.003.

13. Smith S, Dukes-McEwan J. Clinical signs and left atrial size in cats with cardiovascular disease in general practice. J Small Anim Pract. 2012;53(1):2733.

14. Abbott J, MacLean H. Two-dimensional echocardiographic assessment of the feline left atrium. J Vet Intern Med. 2006;20:111-9 Available from: http:// www.ncbi.nlm.nih.gov/pubmed/16496930.

15. Drourr L, Lefbom BK, Rosenthal S, Tyrrell W. Measurement of M-mode echocardiographic parameters in healthy adult Maine Coon cats. J Am Vet Med Assoc [Internet]. 2005;226(5):734-7 Available from: papers2:// publication/uuid/18370688-985C-430D-97A4-6A8465F27812.

16. Soderberg SF, Boon JA, Wingfield WE, Miller CW. M-Mode Echocardiography As a Diagnostic Aid for Feline Cardiomyopathy. Vet Radiol. 1983;24(2):66-73 Available from: http://doi.wiley.com/10.1111/j.1740-8261.1983.tb01541.x.

17. Schille S, Skrodzki M. M-mode echocardiographic reference values in cats in the first three months of life. Vet Radiol Ultrasound [Internet]. 1999;40(5): 491-500 Available from: http://www.ncbi.nlm.nih.gov/pubmed/10528844.

18. Sisson DD, Knight DH, Helinski C, Fox PR, Bond BR, Harpster NK, et al. Plasma taurine concentrations and $\mathrm{M}$-mode echocardiographic measures in healthy cats and in cats with dilated cardiomyopathy. J Vet Intern Med. 1991;5(4):232-8 Available from: http://www.ncbi.nlm.nih.gov/pubmed/1941757.

19. Gavaghan BJ, Kittleson MD, Fisher KJ, Kass PH, Gavaghan MA. Quantification of left ventricular diastolic wall motion by Doppler tissue imaging in healthy cats and cats with cardiomyopathy. Am J Vet Res. 1999;60(12):1478-86.

20. Thomas WP, Gaber CE, Jacobs GJ, Kaplan PM, Lombard CW, Moise NS, et al. Recommendations for standards in transthoracic two-dimensional echocardiography in the dog and cat. Echocardiography Committee of the Specialty of cardiology, American College of Veterinary Internal Medicine. J Vet Intern Med. 1993;7(4):247-52 Available from: http://www.ncbi.nlm.nih. gov/pubmed/8246215.

21. Moise NS, Dietze AE, Mezza LE, Strickland D, Erb HN, Edwards NJ. Echocaridography, electrocardiography, and radiography of cats with dilatation cardiomyopathy, hypertrophic cardiomyopathy, and hyperyroidism. Am J Vet Res. 1986;47(7):1476-86 [cited 2016 Jun 21] Available from: http://inis.iaea.org/Search/search.aspx?orig_q=RN:45107194.

22. Patata V, Caivano D, Porciello F, Rishniw M, Domenech O, Marchesotti F et al. Pulmonary vein to pulmonary artery ratio in healthy and cardiomyopathic cats. J Vet Cardiol. 2020;27:23-33. Available from. https:// doi.org/10.1016/j.jvc.2019.12.001.

23. Mor-Avi V, Yodwut C, Jenkins C, Kühl H, Nesser H-J, Marwick TH, et al. Realtime 3D echocardiographic quantification of left atrial volume. JACC Cardiovasc Imaging [Internet]. 2012;5(8):769-77 Available from: http:// linkinghub.elsevier.com/retrieve/pii/S1936878X12004846.

24. Wesselowski S, Borgarelli M, Bello NM, Abbott J. Discrepancies in Identification of Left Atrial Enlargement Using Left Atrial Volume versus Left Atrial-to-Aortic Root Ratio in Dogs. J Vet Intern Med. 2014;28:1527-33. 
25. Höllmer M, Willesen JL, Tolver A, Koch J. Comparison of four echocardiographic methods to determine left atrial size in dogs. J Vet Cardiol. 2016;18(2):137-45.

26. Duler L, Scollan KF, Leblanc NL. Left atrial size and volume in cats with primary cardiomyopathy with and without congestive heart failure. J Vet Cardiol. 2019;24:36-47. Available from. https://doi.org/10.1016/j.jvc.2019.04. 003.

27. Lang RM, Badano LP, Tsang W, Adams DH, Agricola E, Buck T, et al. GUIDELINES AND STANDARDS EAE / ASE Recommendations for Image Acquisition and Display Using Three-Dimensional. Echocardiography. 2012: 3-46.

28. Nucifora G, Badano LP, Dall'Armellina E, Gianfagna P, Allocca G, Fioretti PM. Fast data acquisition and analysis with real time triplane echocardiography for the assessment of left ventricular size and function: a validation study. Echocardiography. 2009;26(1):66-75

29. Meyer J, Wefstaedt P. Assessment of left ventricular volumes by use of onetwo-, and three-dimensional echocardiography versus magnetic resonance imaging in healthy dogs. Am J Vet Res [Internet]. 2013;10:1223-30 Available from: http://avmajournals.avma.org/doi/abs/10.2460/ajvr.74.9.1223.

30. Buechel RR, Sommer G, Leibundgut G, Rohner A, Riede F, Kessel-Schaefer A, et al. Assessment of left atrial functional parameters using a novel dedicated analysis tool for real-time three-dimensional echocardiography: validation in comparison to magnetic resonance imaging. Int J Cardiovasc Imaging. 2013;29:601-8.

31. Rodevan O, Bjornerheim R, Ljosland M, Maehle J, Smith HJ, Ihlen H. Left atrial volumes assessed by three- and two-dimensional echocardiography compared to MRI estimates. Int J Card Imaging [Internet]. 1999;15(5):397410 Available from: http://eutils.ncbi.n/m.nih.gov/entrez/eutils/elink. fcgi?dbfrom $=$ pubmed\&id $=10595406 \&$ retmode $=$ ref\&cmd $=$ prlinks $\% 5$ Cnpapers3://publication/uuid/B55288D4-AD8E-4D6F-A817-71F8F6A046F1.

32. Tidholm A, Höglund K, Häggström J, Bodegård-Westling A, Ljungvall I. Left atrial ejection fraction assessed by real-time 3-dimensional echocardiography in normal dogs and dogs with myxomatous mitral valve disease. J Vet Intern Med. 2013;27(4):884-9 Available from: http://www.ncbi. nlm.nih.gov/pubmed/23731204.

33. LeBlanc N, Scollan K, Sisson D. Quantitative evaluation of left atrial volume and function by one-dimensional, two-dimensional, and three-dimensional echocardiography in a population of normal dogs. J Vet Cardiol [Internet]. 2016;18(4):336-49. Available from:. https://doi.org/10.1016/j.jvc.2016.06.005.

34. Caballero L, Kou S, Dulgheru R, Gonjilashvili N, Athanassopoulos GD, Barone D, et al. Echocardiographic reference ranges for normal cardiac Doppler data: results from the NORRE study. Eur Heart J Cardiovasc Imaging. 2015; 16(9):1031-41.

35. Russo C, Hahn RT, Jin Z, Homma S, Sacco RL, Di Tullio MR. Comparison of echocardiographic single-plane versus biplane method in the assessment of left atrial volume and validation by real time three-dimensional echocardiography. J Am Soc Echocardiogr [Internet]. 2010;23(9):954-60. Available from:. https://doi.org/10.1016/j.echo.2010.06.010.

36. Vieira-Filho NG, Mancuso FJN, WAA O, Gil MA, Fischer $\mathrm{CH}$, Moises VA. et al. Simplified single plane echocardiography is comparable to conventional biplane two-dimensional echocardiography in the evaluation of left atrial volume: A study validated by three-dimensional echocardiography in 143 individuals. Echocardiography. 2014;31(3):265-72.

37. Caselli S, Canali E, Foschi ML, Santini D, Di Angelantonio E, Pandian NG, et al. Long-term prognostic significance of three- dimensional echocardiographic parameters of the left ventricle and left atrium; 2010. p. 250-6.

38. Maddukuri PV, Vieira MLC, Decastro S, Maron MS, Kuvin JT, Patel AR. What Is the Best Approach for the Assessment of Left Atrial Size ? In: Comparison of Various Unidimensional and Two-dimensional Parameters with Threedimensional Echocardiographically Determined Left Atrial Volume; 2006. p. 1026-32.

39. Ibrahim A, Hager PA, Bartolini A, Angiolini F, Arditi M, Thiran JP, et al. Efficient sample delay calculation for 2-D and 3-D ultrasound imaging. IEEE Trans Biomed Circuits Syst. 2017;11(4):815-31.

40. Boyd AC, Schiller NB, Leung D, Ross DL, Thomas L. Atrial dilation and altered function are mediated by age and diastolic function but not before the eighth decade. JACC Cardiovasc Imaging [Internet]. 2011;4(3):234-42. Available from:. https://doi.org/10.1016/j.jcmg.2010.11.018.

41. Scansen BA, Morgan KL. Reference intervals and allometric scaling of echocardiographic measurements in Bengal cats. J Vet Cardiol [Internet]. 2015;17:S282-95. Available from:. https://doi.org/10.1016/j.jvc.2015.02.001.
42. Häggström J, Andersson A, Falk T, Nilsfors L, Olsson U, Kresken J, et al. Effect of body weight on echocardiographic measurements in 19,866 pure-bred cats with or without heart disease. J Vet Intern Med. 2016;30:1601-11.

43. Schober KE, Stephanie S, Vedat Y. Reference intervals and allometric scaling of two-dimensional echocardiographic measurements in 150 healthy cats. J Vet Med Sci. 2017;79(11):1764-71.

44. Rishniw M, Erb HN. Evaluation of four 2-dimensional echocardiographic methods of assessing left atrial size in dogs. J Vet Intern Med. 2000;14(4): 429-35.

45. Marchesotti F, Vezzosi T, Tognetti R, Marchetti F, Patata V, Contiero B, et al. Left atrial anteroposterior diameter in dogs: reference interval, allometric scaling, and agreement with the left atrial-to-aortic root ratio. J Vet Med Sci. 2019; 10.12(September).

46. Visser LC, Ciccozzi MM, Sintov DJ, Sharpe AN. Echocardiographic quantitation of left heart size and function in 122 healthy dogs : a prospective study proposing reference intervals and assessing repeatability. J Vet Intern Med. 2019;33(January):1909-20.

47. Dickson D, Caivano D, Matos JN, Summerfield N, Rishniw M. Twodimensional echocardiographic estimates of left atrial function in healthy dogs and dogs with myxomatous mitral valve disease. J Vet Cardiol. 2017; 19(6):469-79. Available from. https://doi.org/10.1016/j.jvc.2017.09.003.

48. Leblanc N, Scollan K, Sisson D. Quantitative evaluation of left atrial volume and function by one-dimensional, echocardiography in a population of normal dogs. J Vet Cardiol [Internet]. 2016;18(4):336-49. Available from:. https://doi.org/10.1016/j.jvc.2016.06.005.

49. Horn PS, Pesce AJ. Reference intervals: an update. Clin Chim Acta. 2003; 334(1-2):5-23

50. Friedrichs KR, Harr KE, Freeman KP, Szladovits B, Walton RM, Barnhart KF, et al. ASVCP reference interval guidelines: determination of de novo reference intervals in veterinary species and other related topics. Vet Clin Pathol. 2012;41(4):441-53.

51. Hansson K, Haggstrom J, Kvart C, Lord P. Left atrial to aortic root indices using two-dimensional and $\mathrm{M}$ - mode echocardiography in cavalier King Charles spaniels with and without left atrial enlargement. Vet Radiol Ultrasound [Internet]. 2002;43(6):568-75 Available from: isi:000179786900012

\section{Publisher's Note}

Springer Nature remains neutral with regard to jurisdictional claims in published maps and institutional affiliations.
Ready to submit your research? Choose BMC and benefit from:

- fast, convenient online submission

- thorough peer review by experienced researchers in your field

- rapid publication on acceptance

- support for research data, including large and complex data types

- gold Open Access which fosters wider collaboration and increased citations

- maximum visibility for your research: over $100 \mathrm{M}$ website views per year

At $\mathrm{BMC}$, research is always in progress.

Learn more biomedcentral.com/submissions 